\title{
Randomization and Failure Detection: A Hybrid Approach to Solve Consensus*
}

\author{
Marcos K. Aguilera and Sam Toueg \\ aguilera@cs.cornell.edu sam@cs.cornell.edu \\ Department of Computer Science \\ Upson Hall, Cornell University \\ Ithaca, NY 14853-7501, USA.
}

June 27, 1996

\begin{abstract}
We present a Consensus algorithm that combines randomization and unreliable failure detection, two well-known techniques for solving Consensus in asynchronous systems with crash failures. This hybrid algorithm combines advantages from both approaches: it guarantees deterministic termination if the failure detector is accurate, and probabilistic termination otherwise. In executions with no failures or failure detector mistakes, the most likely ones in practice, Consensus is reached in only two asynchronous rounds.
\end{abstract}

\section{Background}

It is well-known that Consensus cannot be solved in asynchronous systems with failures, even if communication is reliable, at most one process may fail, and it can only fail by crashing. This "impossibility of Consensus", shown in a seminal paper by Fischer, Lynch and Paterson [FLP85], has been the subject of intense research seeking to "circumvent" this negative result (e.g., [Ben83, BT83, Rab83, DDS87, DLS88, CT96, CHT96]).

We focus on two of the major techniques to circumvent the impossibility of Consensus in asynchronous systems: randomization and unreliable failure detection. The first one assumes that each process has an oracle (denoted $R$-oracle) that provides random bits [Ben83]. The second technique assumes that each process has an oracle (denoted $F D$-oracle) that provides a list of processes suspected to have crashed [CT96]. Each approach has some advantages over the other, and we seek to combine advantages from both.

*Research partially supported by NSF grant CCR-9402896 and DARPA/NASA Ames grant NAG-2-593 
With a randomized Consensus algorithm, every process can query its R-oracle, and use the oracle's random bit to determine its next step. With such an algorithm, termination is achieved with probability 1, within a finite expected number of steps (for a survey of randomized Consensus algorithms see [CD89]).

With a failure-detector based Consensus algorithm, every process can query its local FDoracle (which provides a list of processes that are suspected to have crashed) to determine the process's next step. Consensus can be solved with FD-oracles that make an infinite number of mistakes. In particular, Consensus can be solved with any FD-oracle that satisfies two properties, strong completeness and eventual weak accuracy. Roughly speaking, the first property states that every process that crashes is eventually suspected by every correct process, and the second one states that some correct process is eventually not suspected. These properties define the weakest class of failure detectors that can be used to solve Consensus [CHT96].

In this paper we describe a hybrid Consensus algorithm with the following properties. Every process has access to both an R-oracle and an FD-oracle. If the FD-oracle satisfies the above two properties, the algorithm solves Consensus (no matter how the R-oracle behaves). If the FD-oracle loses its accuracy property, but the R-oracle works, the algorithm still solves Consensus, albeit "only" with probability 1. In executions with no failures or failure detector mistakes, the most likely ones in practice, the algorithm reaches Consensus in two asynchronous rounds. A discussion of the relative merits of randomization, failure detection, and this hybrid approach is postponed to Section 7.

The idea of combining randomization and failure detection to solve Consensus in asynchronous systems first appeared in [DM94]. A related idea, namely, combining randomization and deterministic algorithms to solve Consensus in synchronous systems was explored in [GP90, Zam96]. A brief comparison with our results is given in Section 8.

\section{Informal Model}

Our model of asynchronous computation is patterned after the one in [FLP85], and its extension in [CHT96]. We only sketch its main features here. We consider asynchronous distributed systems in which there is no bound on message delay, clock drift, or the time necessary to execute a step. To simplify the presentation of our model, we assume the existence of a discrete global clock. This is merely a fictional device: the processes do not have access to it. We take the range $\mathcal{T}$ of the clock's ticks to be the set of natural numbers $\mathbb{N}$.

The system consists of a set of $n$ processes, $\Pi=\left\{p_{0}, p_{1}, \ldots, p_{n-1}\right\}$. Every pair of processes is connected by a reliable communication channel. Up to $f$ processes can fail by crashing. A failure pattern indicates which processes crash, and when, during an execution. Formally, a failure pattern $F$ is a function from $\mathbb{N}$ to $2^{\Pi}$, where $F(t)$ denotes the set of processes that have crashed through time $t$. Once a process crashes, it does not "recover", i.e., $\forall t$ : $F(t) \subseteq F(t+1)$. We define $\operatorname{crashed}(F)=\bigcup_{t \in \mathbb{N}} F(t)$ and $\operatorname{correct}(F)=\Pi-\operatorname{crashed}(F)$. If $p \in \operatorname{crashed}(F)$ we say $p$ crashes (in $F$ ) and if $p \in \operatorname{correct}(F)$ we say $p$ is correct (in $F$ ).

Each process has access to two oracles: a failure detector, henceforth denoted the $F D$ oracle, and a random number generator, henceforth denoted the $R$-oracle. When a process 
queries its FD-oracle, it obtains a list of processes. When it queries its R-oracle it obtains a bit. The properties of these oracles are described in the two next sections.

A distributed algorithm $\mathcal{A}$ is a collection of $n$ deterministic automata (one for each process in the system) that communicate by sending messages through reliable channels. The execution of $\mathcal{A}$ occurs in steps as follows. For every time $t \in \mathcal{T}$, at most one process takes a step. Each step consists of receiving a message; querying the FD-oracle; querying the R-oracle; changing state; and optionally sending a message to one process. We assume that messages are never lost. That is, if a process does not crash, it eventually receives every message sent to it.

A schedule is a sequence $\left\{s_{j}\right\}_{j \in \mathbb{N}}$ of processes and a sequence $\left\{t_{j}\right\}_{j \in \mathbb{N}}$ of strictly increasing times. A schedule indicates which processes take a step and when: for each $j$, process $s_{j}$ takes a step at time $t_{j}$. A schedule is consistent (with respect to a failure pattern $F$ ) if a process does not take a step after it has crashed (in $F$ ). A schedule is fair (with respect to a failure pattern $F$ ) if each process that is correct (in $F$ ) takes an infinite number of steps. We consider only schedules that are consistent and fair.

\subsection{FD-oracles}

Every process $p$ has access to a local FD-oracle module that outputs a list of processes that are suspected to have crashed. If some process $q$ belongs to such list, we say that $p$ suspects $q .{ }^{1}$ FD-oracles can make mistakes: it is possible for a process $p$ to be suspected by another even though $p$ did not crash, or for a process to crash and never be suspected. FD-oracles can be classified according to properties that limit the extent of such mistakes. We focus on one of the eight classes of FD-oracles defined in [CT96], namely, the class of Eventually Strong failure detectors, denoted $\diamond S$. An FD-oracle belongs to $\diamond S$ if and only if it satisfies two properties:

Strong completeness: Eventually every process that crashes is permanently suspected by every correct process (formally, $\exists t \in \mathcal{T}, \forall p \in \operatorname{crashed}(F), \forall q \in \operatorname{correct}(F), \forall t^{\prime} \geq t$ : $p \in \mathrm{FD}_{q}^{t^{\prime}}$, where $\mathrm{FD}_{q}^{t^{\prime}}$ denotes the output of $q$ 's FD-oracle module at time $\left.t^{\prime}\right)$.

Eventual weak accuracy: There is a time after which some correct process is never suspected by any correct process (formally, $\exists t \in \mathcal{T}, \exists p \in \operatorname{correct}(F), \forall t^{\prime} \geq t, \forall q \in \operatorname{correct}(F)$ : $\left.p \notin \mathrm{FD}_{q}^{t^{\prime}}\right)$.

It is known that $\diamond S$ is the weakest class of FD-oracles that can be used to solve Consensus.

\section{$2.2 \quad$ R-oracles}

Each process has access to a local R-oracle module that outputs one bit each time it is queried. We say that the R-oracle is random if it outputs an independent random bit for each query. For simplicity, we assume a uniform distribution, i.e., a random R-oracle outputs 0 and 1 , each with probability $1 / 2$.

\footnotetext{
${ }^{1}$ In general, processes do not have to agree on the list of suspects at any one time or ever.
} 


\subsection{Adversary Power}

When designing fault-tolerant algorithms, we often assume that an intelligent adversary has some control on the behavior of the system, e.g., the adversary may be able to control the occurrence and the timing of process failures, the message delays, and the scheduling of processes. Adversaries may have limitations on their computing power and on the information that they can obtain from the system. Different algorithms are designed to defeat different types of adversaries [CD89].

We now describe the adversary that our hybrid algorithm defeats. The adversary has unbounded computational power, and full knowledge of all process steps that already occurred. In particular, it knows the contents of all past messages, the internal state of all processes in the system, ${ }^{2}$ and all the previous outputs of both the R-oracle and FD-oracle. With this information, at any time in the execution, the adversary can dynamically select which process takes the next step, which message this process receives (if any), and which processes (if any) crash. The adversary, however, operates under the following restrictions: the final schedule must be consistent and fair, every message sent to a correct process must be eventually received, and at most $f$ processes may crash over the entire execution.

In addition to the above power, we allow the adversary to initially select one of the two oracles to control, and possibly corrupt. ${ }^{3}$ If the adversary selects to control the R-oracle, it can predict and even determine the bits output by that oracle. For example, the adversary can force some local R-oracle module to always output 0 , or it can dynamically adjust the R-oracle's output according to what the processes have done so far.

If the adversary selects to control the FD-oracle, it can ensure that the FD-oracle does not satisfy eventual weak accuracy. In other words, at any time the adversary can include any process (whether correct or not) in the output of the local FD-oracle module of any process. The adversary, however, does not have the power to disrupt the strong completeness property of the FD-oracle. This is not a limitation in practice: most failure detectors are based on time-outs and eventually detect all process crashes.

If the adversary does not control the R-oracle then the R-oracle is random. If the adversary does not control the FD-oracle then the FD-oracle is in $\diamond S$. We stress that the algorithm does not know which one of the two oracles (FD-oracle or R-oracle) is controlled by the adversary.

\section{The Consensus Problem}

Uniform Binary Consensus is defined in terms of two primitives, propose $(v)$ and decide $(v)$, where $v \in\{0,1\}$. When a process executes propose $(v)$, we say that it proposes $v$; similarly, when a process executes decide $(v)$, we say that it decides $v$. The Uniform Binary Consensus problem is specified as follows:

\footnotetext{
${ }^{2}$ This is in contrast to the assumptions made by several algorithms, e.g., those that use cryptographic techniques.

${ }^{3}$ From the definitions of these oracles, it is clear that we can allow the adversary to control the behavior of both oracles for an arbitrary but finite amount of time. The only restriction is that it must eventually stop controlling one of the oracles.
} 
Uniform agreement: If processes $p$ and $p^{\prime}$ decide $v$ and $v^{\prime}$, respectively, then $v=v^{\prime}$;

Uniform validity: If a process decides $v$, then $v$ was proposed by some process;

Termination: Every correct process eventually decides some value.

For probabilistic Consensus algorithms, Termination is weakened to

Termination with probability 1: With probability 1 , every correct process eventually decides some value.

\section{Hybrid Consensus Algorithm}

The hybrid Consensus algorithm shown in Figure 1 combines Ben-Or's algorithm [Ben83] with failure-detection and the rotating coordinator paradigm used in [CT96]. With this paradigm, we assume that all processes have a priori knowledge that during phase $k$, one selected process, namely $p_{k \bmod n}$, is the coordinator. The algorithm works under the assumption that a majority of processes are correct (i.e., $n>2 f$ ). It is easy to see that this requirement is necessary for any algorithm that solves Consensus in asynchronous systems with crash failures, even if all processes have access to a random R-oracle and an FD-oracle that belongs to $\diamond S$.

In the hybrid algorithm, every message contains a tag $(R, P, S$ or $E)$, a phase number, and a value which is either 0 or 1 (for messages tagged $P$ or $S$, it could also be "?"). Messages tagged $R$ are called reports; those tagged with $P$ are called proposals; those with tag $S$ are called suggestions [to the coordinator]; those with tag $E$ are called estimates [from the coordinator]. When $p$ sends $(R, k, v),(P, k, v)$ or $(S, k, v)$ we say that $p$ reports, proposes or suggests $v$ in phase $k$, respectively. When the coordinator sends $(E, k, v)$ we say that the coordinator sends estimate $v$ in phase $k$.

Each execution of the while loop is called a phase, and each phase consists of four asynchronous rounds. In the first round, processes report to each other their current estimate (0 or 1$)$ for a decision value.

In the second round, if a process receives a majority of reports for the same value then it proposes that value to all processes, otherwise it proposes "?". Note that it is impossible for one process to propose 0 and another process to propose 1 . At the end of the second round, if a process receives $f+1$ proposals for the same value different than ?, then it decides that value. If it receives at least one value different than ?, then it adopts that value as its new estimate, otherwise it adopts? for estimate.

In the third round, processes suggest their estimate to the current coordinator. If the coordinator receives a value different than ? then it sends that value as its estimate. Otherwise, the coordinator queries the R-oracle, and sends the random value that it obtains as its estimate.

In the fourth round, processes wait until they receive the coordinator's estimate or until their FD-oracle suspects the coordinator. If a process receives the coordinator's estimate, it 
Every process $p$ executes the following:

0 procedure propose $\left(v_{p}\right)$

$\left\{v_{p}\right.$ is the value proposed by process $\left.p\right\}$

$$
\begin{aligned}
& x \leftarrow v_{p} \\
& k \leftarrow 0
\end{aligned}
$$

while true do

5

$$
k \leftarrow k+1
$$

$c \leftarrow p_{k \bmod n}$

send $(R, k, x)$ to all processes

$\{k$ is the current phase number $\}$ $\{c$ is the current coordinator $\}$

wait for messages of the form $(R, k, *)$ from $n-f$ processes $\{$ " *" can be 0 or 1 \} if received more than $n / 2(R, k, v)$ with the same $v$ then send $(P, k, v)$ to all processes

10 else send $(P, k, ?)$ to all processes

wait for messages of the form $(P, k, *)$ from $n-f$ processes $\{$ "*" can be 0,1 or ?\} if received at least $f+1(P, k, v)$ with the same $v \neq$ ? then $\operatorname{decide}(v)$

if at least one $(P, k, v)$ with $v \neq$ ? then $x \leftarrow v$ else $x \leftarrow$ ?

send $(S, k, x)$ to $c$

15

if $p=c$ then

wait for messages of the form $(S, k, *)$ from $n-f$ processes

if received at least one $(S, k, v)$ with $v \neq$ ?

then send $(E, k, v)$ to all processes

else send $(E, k$, random_bit) to all processes

wait until receive $\left(E, k, v_{-}\right.$coord $)$from $c$ or $c \in F D$-oracle \{query FD-oracle\} if received $\left(E, k, v_{-}\right.$coord $)$

then $x \leftarrow v_{\text {_coord }}$ 
adopts it. Otherwise, if its current estimate is ?, it adopts a random value obtained from its R-oracle.

To simplify the presentation, the algorithm in Figure 1 does not include a halt statement. Moreover, once a correct process decides a value, it will keep deciding the same value in all subsequent phases. However, it is easy to modify the algorithm so that every process decides at most once, and halts at most one round after deciding.

\section{Proof of Correctness}

The hybrid algorithm shown in Figure 1 always satisfies the safety properties of Consensus. This holds no matter how the FD-oracle or the R-oracle behave, that is, even if these oracles are totally under the control of the adversary. On the other hand, the algorithm satisfies liveness properties only if the FD-oracle satisfies strong completeness. Strong completeness is easy to achieve in practice: most failure-detectors use time-out mechanisms, and every process that crashes eventually causes a time-out, and therefore a permanent suspicion.

Assume that there is a majority of correct processes (i.e., $n>2 f$ ). We show the following:

\section{Theorem 1}

(Safety) The hybrid algorithm always satisfies validity and uniform agreement.

(Liveness) Suppose that the FD-oracle satisfies strong completeness.

- If the FD-oracle satisfies eventual weak accuracy, i.e., it is in $\diamond S$, then the algorithm satisfies termination.

- If the $\mathrm{R}$-oracle is random then the algorithm satisfies termination with probability 1.

Proof: We say that process $p$ starts phase $k$ if process $p$ completes at least $k-1$ iterations of the while loop. We say that process $p$ reaches line $n$ in phase $k$ if process $p$ starts phase $k$ and $p$ executes past line $n-1$ in that phase. We say that $v$ is $k$-locked if every process that starts phase $k$ does so with its variable $x$ set to $v$. When ambiguities may arise, a local variable of a process $p$ is subscripted by $p$, e.g., $x_{p}$ is the local variable $x$ of process $p$.

We first show the safety properties.

Lemma 1 Suppose $k>0$. Then (1) it is impossible for a process to propose 0 and another one to propose 1 in the same phase $k$; and (2) it is impossible for a process to suggest 0 and another to suggest 1 in the same phase $k$.

Proof: We prove (1) by contradiction: suppose that two processes $p$ and $q$ propose 0 and 1 , respectively, in phase $k$. Thus, $p$ received more than $n / 2$ reports for 0 and $q$ received more than $n / 2$ reports for 1 in phase $k$. But then there is a process that reports 0 to $p$ and 1 to $q$ in phase $k$, and this is impossible. This proves (1).

Now (2) follows from (1) since if a process suggests $v \neq$ ? in phase $k$, then $v$ was proposed in phase $k$. 
Lemma 2 If some process decides $v$ in phase $k>0$, then $v$ is $(k+1)$-locked.

Proof: Suppose some process $p$ decides $v$ in phase $k>0$ (note that $v \neq ?$ ), and let $q$ be any process that starts phase $k+1$. From the algorithm, $p$ receives at least $f+1$ proposals for $v$ in phase $k$ (line 12). Let $r$ be any process that suggests a value in line 14 of phase $k$. Before suggesting (line 14), $r$ waits for $n-f$ proposals in line 11. Because $p$ receives $f+1$ proposals for $v, r$ must have received at least one proposal for $v$. Moreover, by Lemma $1, r$ does not receive any proposals for $\bar{v}$. ${ }^{4}$ So $r$ sets $x_{r}$ to $v$ in line 13 and suggests $v$ in phase $k$. Thus, (1) $q$ sets $x_{q}$ to $v$ in line 13 , and (2) the coordinator of phase $k$ can only receive suggestions for $v$. In particular, the coordinator does not receive ?. So, if the coordinator sends an estimate in phase $k$ (line 18), that estimate is also $v$. If $q$ receives that estimate (line 22), then $q$ resets $x_{q}$ to $v$ in line 24. Otherwise $q$ does not modify $x_{q}$ (because $x_{q}$ is different than ?). In either case, $q$ starts phase $k+1$ with $x_{q}=v$.

Lemma 3 If a value $v$ is $k$-locked for some $k>0$, then every process that reaches line 13 in phase $k$ decides $v$ in phase $k$.

Proof: Suppose $v$ is $k$-locked for some $k>0$. Then, all reports sent in line 6 of phase $k$ are for $v$. Since $n-f>n / 2$, every process that proposes some value in phase $k$ proposes $v$ in line 9 . Consider a process $p$ that reaches line 13 in phase $k$. Clearly, $p$ receives $n-f$ proposals (line 11) for $v$ in phase $k$. Since $n-f \geq f+1, p$ decides $v$ in phase $k$.

Corollary 1 If some process decides $v$ in phase $k>0$, then every process that reaches line 13 in phase $k+1$ decides $v$ in phase $k+1$.

Proof: By Lemma 2 and Lemma 3.

Corollary 2 (Uniform agreement) If some processes $p$ and $p^{\prime}$ decide $v$ and $v^{\prime}$ in phase $k>0$ and $k^{\prime}>0$, respectively, then $v=v^{\prime}$.

Proof: For $k=k^{\prime}$ the result follows from Lemma 1 and the fact that a process can decide a value in a phase only if that value was proposed in the same phase. Assume that $k<k^{\prime}$. Since $p^{\prime}$ decides in phase $k^{\prime}$ then $p^{\prime}$ reaches line 13 in every phase $r, k<r \leq k^{\prime}$. Since $p$ decides $v$ in phase $k$, by Corollary $1 p^{\prime}$ decides $v$ in phase $k+1 \leq k^{\prime}$. By additional applications of Corollary 1 , we conclude that $p^{\prime}$ decides $v$ in phase $k^{\prime}$. Each process can decide at most once per phase, so $v=v^{\prime}$.

Corollary 3 (Validity) If some process $p$ decides some value $v$ then $v$ was the initial value of some process.

Proof: Note $v \in\{0,1\}$. If the initial values of all processes are not identical, then $v$ is clearly the initial value of some process. Now, suppose all processes have the same initial value $w$. Thus, $w$ is 1-locked. From Lemma 3,p decides $w$, and from Corollary $2, w=v$.

From now on we assume that the FD-oracle satisfies strong completeness, and proceed to prove the liveness properties.

\footnotetext{
${ }^{4}$ We denote by $\bar{v}$ the binary complement of bit $v$.
} 
Lemma 4 Every correct process starts every phase $k>0$.

Proof: The detailed proof is by a simple but tedious induction on $k$. We describe only the central idea here. In each phase, there are four wait statements that can potentially block processes (lines 7, 11, 16, 22). It is not possible for a correct process to be blocked forever in any of the first three wait statements, because at least $n-f$ processes are correct and send the messages that this process is waiting for. Consider the fourth wait statement. Either the coordinator $c$ sends its estimate to all processes or $c$ crashes. In the first case, every correct process receives this estimate. In the second case, $c$ eventually appears on the list of suspects, i.e., $c \in F D$-oracle (because the $F D$-oracle satisfies strong completeness). So no correct process waits forever at the fourth wait statement of a phase.

Corollary 4 If a value $v$ is $k$-locked for some $k>0$, then every correct process decides $v$ in phase $k$.

Proof: Immediate from Lemmata 3 and 4.

Corollary 5 If some process decides $v$ in phase $k>0$, then every correct process decides $v$ in phase $k+1$ (and thus in all subsequent phases).

Proof: Immediate from Corollary 1 and Lemma 4.

Lemma 5 (Termination) If the FD-oracle satisfies eventual weak accuracy then every correct process decides.

Proof: By eventual weak accuracy of the FD-oracle, there exists a time after which a correct process $p_{m}$ is never suspected by anyone. For each process $p_{i}$, let $k_{i}$ be the value of variable $k$ of process $p_{i}$ at that time. Let $\bar{k}=1+\max _{i}\left\{k_{i}\right\}$ and $\hat{k}=\min \{j+\bar{k}: j \in \mathbb{N} \wedge(j+\bar{k}) \bmod n=m\}$. Let $q$ and $r$ be arbitrary processes that start phase $\hat{k}+1$. In phase $\hat{k}$, the coordinator is process $p_{m}$, by choice of $\hat{k}$. So $q$ and $r$ do not suspect the coordinator and thus $q$ and $r$ set $x_{q}$ and $x_{r}$ to the coordinator's estimate in line 24. Since this estimate is different from ? and unique for phase $\hat{k}$, we have $x_{q}=x_{r}=v$ for some $v \neq$ ? at the beginning of phase $\hat{k}+1$. So $v$ is $\hat{k}+1$-locked. Therefore, by Corollary 4 , all correct processes decide $v$ in phase $\hat{k}+1$.

We now proceed to show that if the R-oracle is random, then the algorithm satisfies termination with probability 1 . For $k>0$, let $\tau_{k}$ be the first time that any process receives $n-f$ proposals in phase $k$. From Lemma 4 , for every $k>0$, some process receives $n-f$ proposals in phase $k$, and so $\tau_{k}$ is well-defined. Note that in our algorithm no process queries the R-oracle in phase $k$ before time $\tau_{k}$.

For each $k>0$, we say that a value $v$ is $k$-major at time $t$ if by time $t$ more than $n / 2$ processes have started phase $k$ with their variable $x$ set to $v$. Clearly, for each $k>0$ and all times $t$ and $t^{\prime}$, it is impossible for 0 to be $k$-major at $t$, and 1 to be $k$-major at $t^{\prime}$.

We say that a process $p$ R-gets $v$ in phase $k$ at time $t$ if either:

- In phase $k$ at time $t, p$ obtains $v$ from the R-oracle in line 25 and sets $x_{p}$ to $v$; or

- In phase $k$, the coordinator obtains $v$ from the R-oracle in line 20 , sends $v$ as its estimate to all processes, $p$ receives this estimate and sets $x_{p}$ to $v$ in line 24 at time $t$. 
function FavorableToss $(r, u)$ : bit

$k \leftarrow 2 r$ \{evaluated only at time $u \geq \tau_{k}$ where $k=2 r$ \}

$\{k$ is the first phase in epoch $r\}$

if some value $v$ is $k$-major at time $\tau_{k}$ then return $v$

if by time $u$ no process received $n-f$ proposals in phase $k+1$ then return $0\left\{u<\tau_{k+1}\right\}$

if before time $\tau_{k+1}$ :

\{here $\left.u \geq \tau_{k+1}\right\}$

(a) 1 is $k$-major, and

(b) less than $n / 2$ processes $\mathrm{R}$-got a value in phase $k$, and

(c) the coordinator did not query the R-oracle in line 20 of phase $k$

then return 1

else return 0

Figure 2: Favorable coin toss algorithm

Intuitively, a process $p$ R-gets $v$ if $p$ sets $x_{p}$ to $v$, and $p$ obtained $v$ from an R-oracle query (directly, or indirectly through the coordinator).

Lemma 6 If at time $t$ a process $p$ starts phase $k>1$ with $x_{p}$ set to some value $v$, then either $v$ is $(k-1)$-major at time $t$ or $p$ R-gets $v$ in phase $k-1$.

Proof: Consider phase $k-1$. Suppose $p$ did not R-get $v$. Let $t^{\prime}$ be the last time $p$ updates $x_{p}$ in phase $k-1$. Note that $t^{\prime}<t$. Then, at time $t^{\prime}$, either (a) $p$ receives the estimate from the coordinator, and the coordinator obtained that estimate from one of its non-? suggestions; or (b) $p$ sets $x_{p}$ in line 13 . In both cases, more than $n / 2$ processes must have reported $v$ in phase $k-1$ before time $t^{\prime}$. Therefore, at least $n / 2$ processes have started phase $k-1$ by time $t^{\prime}$ (and thus by time $t$ ) with their variable $x$ set to $v$.

For the rest of the proof, we group pairs of phases into epochs as follows: epoch $r$ consists of phases $2 r$ and $2 r+1 .{ }^{5}$ We will define the concept of a "lucky" epoch - one in which processes toss coins that cause the termination of the algorithm (no matter what the adversary does). To do so, we first define function FavorableToss $(r, u)$ given in Figure 2. We say that epoch $r$ is lucky if, for every process $p$ and any time $u$, if $p$ queries the R-oracle in epoch $r$ at time $u$, then $p$ obtains FavorableToss $(r, u)$ from the R-oracle. Note that if $p$ queries the R-oracle in epoch $r$ at time $u$, this occurs after at least one process receives $n-f$ proposals in phase $2 r$. Thus, $\tau_{2 r} \leq u$, so the value of FavorableToss $(r, u)$ depends only on what occurred in the system up to time $u$.

Lemma 7 If the R-oracle is random then the probability that some epoch is lucky is 1.

Proof: The result is immediate from the following observation: for every $r \geq 1$, (a) the probability that epoch $r$ is lucky is at least $2^{-(2 n+2)}$ (because in each phase there are at most

\footnotetext{
${ }^{5}$ Phase 1 is not part of any epoch.
} 
$n+1$ queries to the R-oracle, and the R-oracle is random), and (b) for any $r^{\prime} \neq r$, the events "epoch $r$ is lucky" and "epoch $r^{\prime}$ is lucky" are independent (because epochs $r$ and $r^{\prime}$ consist of disjoint sets of phases).

Lemma 8 For every $r \geq 1$, if epoch $r$ is lucky then some value is $(2 r+1)$-locked or $(2 r+2)$-locked.

Proof: Throughout the proof of this lemma, fix some arbitrary $r \geq 1$ and assume that epoch $r$ is lucky. Let $k=2 r$; recall that epoch $r$ consists of phases $k$ and $k+1$. Since epoch $r$ is lucky, if any process R-gets a value $v$ at time $t$ and in phase $j=k$ or $j=k+1$, then $v=$ FavorableToss $(r, u)$ for some time $u, \tau_{j} \leq u \leq t$ (value $v$ was obtained either directly from the R-oracle or indirectly through the coordinator).

Case 1: Suppose some value $v$ is $k$-major at time $\tau_{k}$. By the definition of FavorableToss, for any $u$ such that $\tau_{k} \leq u$, FavorableToss $(r, u)=v$. So, if a process R-gets a value in phase $k$, that value is $v$. Note that $\bar{v}$ is not $k$-major at any time. By Lemma $6, v$ is $(k+1)$-locked.

Case 2: Now assume that no value is $k$-major at time $\tau_{k}$.

Case 2.1: Suppose that no value is $k$-major before time $\tau_{k+1}$. Then for any $u, \tau_{k} \leq u$, we have FavorableToss $(r, u)=0$. By Lemma 6 , every process $p$ that starts phase $k+1$ before time $\tau_{k+1}$ does so with $x_{p}$ set to some value that $p$ R-got in phase $k$, and such value can only be 0 . So all reports (and thus all proposals) sent in phase $k+1$ before time $\tau_{k+1}$ are for 0 . From the definition of $\tau_{k+1}$, there are at least $n-f$ such proposals for 0 in phase $k+1$. By an argument similar to the one in the proof of Lemma 2, value 0 is $(k+2)$-locked.

Case 2.2: Now assume some value $v$ is $k$-major before time $\tau_{k+1}$.

Case 2.2.1: Suppose $v=0$. Since 1 is never $k$-major, then for any time $u$ such that $\tau_{k} \leq u$, we have FavorableToss $(r, u)=0$. So all processes that $\mathrm{R}$-get a value in phase $k \mathrm{R}$-get 0 . By Lemma 6, value 0 is $(k+1)$-locked.

Case 2.2.2: Now assume $v=1$. For any time $u, \tau_{k} \leq u<\tau_{k+1}$, we have FavorableToss $(r, u)=0$. Let $S$ be the processes that R-get a value in phase $k$ before time $\tau_{k+1}$; clearly, all processes in $S$ R-get 0 .

Case 2.2.2.1: Suppose $|S| \geq n / 2$. Then for any time $u, \tau_{k} \leq u$, FavorableToss $(r, u)=0$. So, all processes that R-get in phase $k+1$ R-get 0 . Note that $|S| \geq n / 2$ implies that 1 can never be $(k+1)$-major. By Lemma 6 , value 0 is $(k+2)$-locked.

Case 2.2.2.2: Now assume that $|S|<n / 2$.

Case 2.2.2.2.1: Suppose that the coordinator of phase $k$ does not query the R-oracle in line 20 of phase $k$ before time $\tau_{k+1}$. Then for any $u$ such that $\tau_{k+1} \leq u$, we have FavorableToss $(r, u)=1$. So, if the coordinator queries the R-oracle in line 20 of 
phase $k$ it obtains 1 from the R-oracle. Therefore, all processes that $\mathrm{R}$-get a value at or after time $\tau_{k+1}$ in phase $k$ R-get 1 . Thus, exactly $|S|<n / 2$ processes R-get 0 in phase $k$. Since 1 is $k$-major, from Lemma 6 we conclude that value 0 can never be $(k+1)$-major. Since no process queries the R-oracle in phase $k+1$ before time $\tau_{k+1}$, all processes that $\mathrm{R}$-get a value in phase $k+1$ R-get 1 . By Lemma 6 , value 1 is $(k+2)$-locked.

Case 2.2.2.2.2: Now assume that the coordinator of phase $k$ queries the R-oracle in line 20 of phase $k$ before time $\tau_{k+1}$. Then the coordinator obtains 0 from the R-oracle. So, for any $u \geq \tau_{k}$, we have FavorableToss $(r, u)=0$. Since the coordinator queries the R-oracle in line 20, it received $n-f$ suggestions for ? in line 16, and this occurred before time $\tau_{k+1}$. Thus, $n-f$ processes have set their variable $x$ to $?$ in line 13 in phase $k$ before time $\tau_{k+1}$. Note that if any such process starts phase $k+1$, then it R-gets a value in phase $k$, and that value is 0 , and thus such process starts phase $k+1$ with its variable $x$ set to 0 . Therefore at most $n-(n-f)=f<n / 2$ processes start phase $k+1$ with their variable $x$ set to 1 . So 1 can never be $(k+1)$-major. All processes that $\mathrm{R}$-get in phase $k+1 \mathrm{R}$-get 0 . By Lemma 6 , value 0 is $(k+2)$-locked. $\square$

Lemma 9 (Termination with probability 1) If the $R$-oracle is random then the probability that all correct processes decide is 1 .

Proof: Immediate from Lemmata 7 and 8, and Corollary $4 . \square$

The proof of Theorem 1 is now complete: validity and uniform agreement were shown in Corollary 3 and Corollary 2, respectively. Termination was proved in Lemma 5, and termination with probability 1 was shown in Lemma 9.

$\square_{\text {Theorem } 1}$

From the proof of Lemma 7, it is easy to see that the expected number of rounds for termination is $O\left(2^{2 n}\right)$. However, it can be shown that, as in [Ben83], termination is reached in constant expected number of rounds if $f=O(\sqrt{n})$. In Section 7 , we outline a similar hybrid algorithm that terminates in constant expected number of rounds even for $f=O(n)$.

\section{An Optimization}

The algorithm in Figure 1 was designed to be simple rather than efficient, because our main goal here is to demonstrate the viability of a "robust" hybrid approach (one in which termination can occur in more than one way: by "good" failure detection or by "good" random draws). The following optimization suggests that such hybrid algorithms can also be efficient in practice.

In many systems, failures are rare, and failure detectors can be tuned to seldom make mistakes (i.e., erroneous suspicions). The algorithm in Figure 1 can be optimized to perform particularly well in such systems. The optimized version ensures that all correct processes decide by the end of two asynchronous rounds when the first coordinator does not crash and no process erroneously suspects it. ${ }^{6}$

\footnotetext{
${ }^{6}$ Actually, decision occurs in two rounds even if up to $n-2 f-1$ processes erroneously suspect it.
} 
$c \leftarrow p_{0}$

if $p=c$ then send $\left(E, 0, v_{p}\right)$ to all processes

$\left\{p_{0}\right.$ is the first coordinator $\}$

\{if $p$ is the first coordinator

wait until receive $\left(E, 0, v_{-}\right.$coord $)$from $c$ or $c \in F D$-oracle

\{query FD-oracle\}

if received $\left(E, 0, v_{-}\right.$coord $)$

then send $\left(P, 0, v_{-}\right.$coord $)$to all processes

else send $(P, 0, ?)$ to all processes

wait for messages of the form $(P, 0, *)$ from $n-f$ processes $\quad\{$ "* can be 0,1 or ?\} if received at least $f+1(P, 0, v)$ with the same $v \neq$ ? then $\operatorname{decide}(v)$

if received at least one $(P, 0, v)$ with $v \neq$ ? then $x \leftarrow v$

Figure 3: Optimization for the hybrid algorithm

This optimization is obtained by inserting some extra code between lines 2 and 3 of the hybrid algorithm. This code, given in Figure 3, consists of a phase (phase 0) with two asynchronous rounds. In the first round, $p_{0}$ sends a message to all processes; in the second round, every process sends a message to all processes. We claim that: (1) the optimization code preserves the correctness of the original algorithm; and (2) processes decide quickly in the absence of failures and erroneous suspicions. To see (1) note that:

- No correct process blocks during the execution of the optimization code (phase 0), i.e., all correct processes start phase 1 ;

- Any process $p$ that starts phase 1 does so with $x_{p}$ set to the initial value of some process;

- If some process decides $v$ in phase 0 then $v$ is 1-locked. Thus, (by Corollary 4) all correct processes decide $v$ in phase 1 .

To see (2), note that if $p_{0}$ is correct and no process suspects $p_{0}$, then all processes wait for its estimate $v$ and propose $v$ in phase 0 ; so every process receives $n-f$ proposals for $v$ and thus decides $v$ in phase 0 . Thus we have:

Theorem 2 Theorem 1 holds for the optimized hybrid algorithm. Moreover, in executions with no crashes or false suspicions, all processes decide in two rounds.

\section{Discussion}

In practice, many systems are well-behaved most of the time: few failures actually occur, and most messages are received within some predictable time. Failure-detector based algorithms (whether "pure" ones like in [CT96] or hybrid ones like in this paper) are particularly wellsuited to take advantage of this: (time-out based) failure detectors can be tuned so that 
the algorithms perform optimally when the system behaves as predicted, and performance degrades gracefully as the system deviates from its "normal" behavior (i.e., if failures occur or messages take longer than expected). For example, the optimized version of our hybrid algorithm solves Consensus in only two asynchronous rounds in the executions that are most likely to occur in practice, namely, runs with no failures or erroneous suspicions.

The above discussion suggests that using this hybrid approach is better than using the randomized approach alone. In fact, randomized Consensus algorithms for asynchronous systems tend to be inefficient in practical settings. ${ }^{7}$ Typically, their performance depends more on "luck" (e.g., many processes happen to start with the same initial value or happen to draw the same random bit) than on how "well-behaved" the underlying system is (e.g., on the number of failures that actually occur during execution). The fact that randomized algorithms are extremely "robust", i.e., they do not depend on how the system behaves, may also be an inherent source of inefficiency.

Note that our hybrid algorithm terminates with probability 1 even if the FD-oracle is completely inaccurate (in fact even if every process suspects every other process all the time). So it is more robust than algorithms that are simply failure-detector based.

An important remark is now in order about the expected termination time of our hybrid algorithm. We developed this algorithm by combining Ben-Or's randomized algorithm [Ben83] with the failure detection ideas in [CT96]. We selected Ben-Or's algorithm because it is the simplest, and thus the most appropriate to illustrate this approach, even though its expected number of rounds is exponential in $n$ for $f=O(n)$. By starting from an efficient randomized algorithm, due to Chor et al. [CMS89], we can obtain a hybrid algorithm that terminates in constant expected number of rounds, as we now briefly explain.

Roughly speaking, the randomized asynchronous Consensus algorithm in [CMS89] is obtained from Ben-Or's algorithm by replacing each coin toss with the toss of a "weakly global coin" computed by a coin_toss procedure. We can do exactly the same: replace the coin tosses of the algorithm in Figure 1 with those obtained by using the coin_toss procedure. More precisely, in each phase, every process: (a) invokes this procedure between the second and third rounds (i.e., between lines 13 and 14) to obtain a random bit, and (b) uses this random bit rather than querying the R-oracle (in lines 20 and 25 ). ${ }^{8}$

As in [CMS89], this modified hybrid algorithm terminates ${ }^{9}$ in constant expected number of rounds for $f \leq n(3-\sqrt{5}) / 2 \approx 0.38 n$. But also as in [CMS89], and in contrast to the algorithm in Section 4, it assumes that the adversary cannot see the internal state of processes or the content of messages. With the optimization of Figure 3, this modified hybrid algorithm also terminates in two rounds in failure-free and suspicion-free runs.

\footnotetext{
${ }^{7}$ Algorithms that assume that processes a priori agree on a long sequence of random bits [Rab83, Tou84] are more efficient than others. But this assumption may be too strong for some systems.

${ }^{8} \mathrm{As}$ in [CMS89], another simple modification is necessary: the addition of a "synchronization round" just before the coin_toss procedure. In this round, processes broadcast "wait" messages, then wait until $n-f$ such messages are received.

${ }^{9}$ Provided, of course, that the FD-oracle satisfies strong completeness.
} 


\section{Related Work}

The idea of combining randomization with a deterministic Consensus algorithm appeared in [GP90], and was further developed in [Zam96]. These works, however, are for synchronous systems only and do not involve failure detection.

Dolev and Malki were the first to combine randomization and unreliable failure detection to solve Consensus in asynchronous systems with process crashes [DM94]. That work differs from ours in many aspects:

- The hybrid algorithms given in [DM94] assume that both the R-oracle and the FDoracle always work correctly. If the failure detector loses it accuracy property, processes may decide differently; if the random source of bits is corrupted, processes may never decide.

- Two goals of [DM94] are to use failure detection to increase the resiliency and ensure the deterministic termination of randomized Consensus algorithms. The hybrid Consensus algorithms given in [DM94] achieve the first goal, by increasing the resiliency from $f<n / 2$ to $f<n$, but not the second one. It is stated, however, that a future version of the paper will give an algorithm that achieves both goals.

- The two hybrid algorithms in [DM94] use failure detectors that are stronger than $\diamond S$. The first one - which supposes that the same sequence of random bits is shared by all the processes, as in [Rab83] — assumes that some correct process is never suspected by any process. The second algorithm - which drops the assumption of a common sequence of bits - assumes that $\Omega(n)$ correct processes are never suspected by any process.

\section{Acknowledgement}

We are grateful to Vassos Hadzilacos: some of our proofs are based on his lecture notes. We would also like to thank the anonymous referees for their valuable comments.

\section{References}

[Ben83] Michael Ben-Or. Another advantage of free choice: Completely asynchronous agreement protocols. In Proceedings of the Second ACM Symposium on Principles of Distributed Computing, pages 27-30, August 1983.

[BT83] Gabriel Bracha and Sam Toueg. Resilient consensus protocols. In Proceedings of the Second ACM Symposium on Principles of Distributed Computing, pages 1226, August 1983. An extended and revised version appeared as "Asynchronous consensus and broadcast protocols" in the Journal of the ACM, 32(4):824-840, October 1985. 
[CD89] Benny Chor and Cynthia Dwork. Randomization in Byzantine Agreement. Advances in Computer Research (JAI Press Inc.), 4:443-497, 1989.

[CHT92] Tushar Deepak Chandra, Vassos Hadzilacos, and Sam Toueg. The weakest failure detector for solving consensus. In Proceedings of the Tenth ACM Symposium on Principles of Distributed Computing, pages 147-158, August 1992.

[CHT96] Tushar Deepak Chandra, Vassos Hadzilacos, and Sam Toueg. The weakest failure detector for solving consensus. Journal of the ACM, 43(4), July 1996. An earlier version appeared in [CHT92].

[CMS89] Benny Chor, Michael Merritt, and David B. Shmoys. Simple constant-time consensus protocols in realistic failure models. Journal of the ACM, 36(3):591-614, 1989.

[CT91] Tushar Deepak Chandra and Sam Toueg. Unreliable failure detectors for asynchronous systems. In Proceedings of the Tenth ACM Symposium on Principles of Distributed Computing, pages 325-340. ACM Press, August 1991.

[CT96] Tushar Deepak Chandra and Sam Toueg. Unreliable failure detectors for reliable distributed systems. Journal of the ACM, 43(2):225-267, March 1996. An earlier version appeared in [CT91].

[DDS87] Danny Dolev, Cynthia Dwork, and Larry Stockmeyer. On the minimal synchronism needed for distributed consensus. Journal of the ACM, 34(1):77-97, January 1987.

[DLS88] Cynthia Dwork, Nancy A. Lynch, and Larry Stockmeyer. Consensus in the presence of partial synchrony. Journal of the ACM, 35(2):288-323, April 1988.

[DM94] Danny Dolev and Dalia Malki. Consensus made practical. Technical Report CS94-7, The Hebrew University of Jerusalem, March 1994.

[FLP85] Michael J. Fischer, Nancy A. Lynch, and Michael S. Paterson. Impossibility of distributed consensus with one faulty process. Journal of the ACM, 32(2):374382, April 1985.

[GP90] Oded Goldreich and Erez Petrank. The best of both worlds: guaranteeing termination in fast randomized Byzantine Agreement protocols. Information Processing Letters, 36(1):45-49, October 1990.

[Rab83] Michael Rabin. Randomized Byzantine Generals. In Proceedings of the TwentyFourth Symposium on Foundations of Computer Science, pages 403-409. IEEE Computer Society Press, November 1983.

[Tou84] Sam Toueg. Randomized Byzantine Agreements. In Proceedings of the Third ACM Symposium on Principles of Distributed Computing, pages 163-178, August 1984. 
[Zam96] Arkady Zamsky. A randomized Byzantine Agreement protocol with constant expected time and guaranteed termination in optimal (deterministic) time. In Proceedings of the Fifteenth ACM Symposium on Principles of Distributed Computing, pages 201-208, May 1996. 